9th A. Friedmann International Seminar and

3rd Casimir Symposium 2015

International Journal of Modern Physics: Conference Series

Vol. 41 (2016) 1660140 (8 pages)

(C) The Author(s)

DOI: $10.1142 / \mathrm{S} 201019451660140 \mathrm{X}$

\title{
Short-scale atomic clock based on the quantum magnetometers system
}

\author{
S. V. Ermak \\ Institute of Physics, Nanotechnology and Telecommunications, \\ Peter the Great Saint Petersburg Polytechnic University, \\ 29 Politekhnicheskaya street, Saint Petersburg, 195251, Russian Federation \\ serge_ermak@mail.ru \\ E. A. Sagitov \\ Institute of Physics, Nanotechnology and Telecommunications, \\ Peter the Great Saint Petersburg Polytechnic University, \\ 29 Politekhnicheskaya street, Saint Petersburg, 195251, Russian Federation \\ e-sagitov@mail.ru \\ R. V. Smolin \\ Institute of Physics, Nanotechnology and Telecommunications, \\ Peter the Great Saint Petersburg Polytechnic University, \\ 29 Politekhnicheskaya street, Saint Petersburg, 195251, Russian Federation \\ smolin_roman@inbox.ru \\ V. V. Semenov \\ Institute of Physics, Nanotechnology and Telecommunications, \\ Peter the Great Saint Petersburg Polytechnic University, \\ 29 Politekhnicheskaya street, Saint Petersburg, 195251, Russian Federation \\ vladimir_semenov@mail.ru
}

Received 24 September 2015

Revised 29 September 2015

Published 18 March 2016

\begin{abstract}
The experimental results of dependence of Allan variance as averaging time for system of two quantum magnetometers with laser pumping of the alkaline atoms are presented. Also the role of different components of luminous frequency shift of a radio optical resonance in case of an optimum operation mode of quantum magnetometers in such system is noted. It is shown that the effect of compensation of luminous shift components
\end{abstract}

This is an Open Access article published by World Scientific Publishing Company. It is distributed under the terms of the Creative Commons Attribution 4.0 (CC-BY) License. Further distribution of this work is permitted, provided the original work is properly cited. 
allows to reduce Allan variance in times of averaging more, than hundreds of seconds in comparison with the quantum standard of frequency on 0-0 transition.

Keywords: Optical pumping; vapor cells; quantum magnetometer; Allan variance.

PACS numbers: 42.50.Gy, 06.30.Ft

\section{Introduction}

Atomic Clocks implement the synchronization of telecommunication and precise time systems. They ensures correct operations of stationary and aerospace-based manned and unmanned systems, ensuring their reliability and accuracy. The basis for the Atomic Clock constructing is the optical pumping method, have been developed since the beginning of the 50 s of XX century. ${ }^{1}$ The optical pumping leads to the redistribution of populations in the atom energy structure ground state, that creates the conditions to monitor the magneto-dipole transitions in the ultra-fine structure (UFS), providing precision measurement of the resonance frequency, that determine the stability of portable atomic clock. ${ }^{2}$ The main measurement error source are the light frequency shift associated with the influence of non-resonant light components. ${ }^{3}$ Theoretical and experimental researches have shown the ability to reduce the influence of the light frequency shift component compensation in the case of end-state magneto-dependent UFS-transitions (end-state resonance), which is not possible in the case of magneto-dependent 0-0-transition. ${ }^{4}$ In the case of the atomic clock on end-state resonance, Zeeman transitions self-oscillating magnetometer can be selected to compensate the magnetic fluctuations in an external magnetic field. Hereby, the compensation of the magneto-dependent UFS end-state resonance magnetic dependence and the additional frequency destabilizing factors of the atomic clock is provided. ${ }^{5}$ Practically, this compensation achieved by simultaneous signal registration of end-state UFS resonance using the technique of synchronous detection and of self-oscillating quantum magnetometer. These oscillation are on the magneto-dipole transitions average frequency of the ${ }^{87} \mathrm{Rb}$ atoms ground state. The further processing of the received signals are provided. Thus, the frequency light shifts compensation is very impotent for atomic clock long term stability achievement, and is learning in this article. The effect of the light shift component compensation is demonstrated. To achieve this objective it is necessary to solve following problems:

1. The experimental research of the end-state UFS-resonance frequency stability in laser pumped ${ }^{87} \mathrm{Rb}$ atoms, determination of Allan variance and its subsequent analysis;

2. The development of the compensation destabilizing factors methods using the signals of two magnetometers;

3. The elaboration of the recommendations for optical pumping modes optimization in alkaline atoms placed into the absorption cell with wall anti-relaxation coating and allowing to reduce frequency errors of the atomic clock. 


\section{The Method of Optical Pumping}

Main task is the creation of non-equilibrium population of atoms in the energetic structure. It achieves by method of optical pumping, which is widely used in modern devices of quantum electronics and primarily includes exemplary frequency measurements and a whole range of precision quantum magnetometers used in various technical applications, and in a study of fine physical phenomena. The basic substance of such devices are alkali metal atoms.

The object of optical pumping in this energy structure is the ground state, where in thermodynamic equilibrium conditions all magnetic sublevels have equilibrium populations. Optical pumping, i.e. the resonant light effect on the atoms, leads to the inversion of the populations in the ground state, that creates conditions for monitoring magneto-dipole transitions in ultra-fine structure.

Until recently, the technique of optical pumping have used spectral lamps filled with the same matter as the pumping atoms. With the progress in development of the laser sources there is a great interest to use laser pumping in various alkali metals to produce wide range of quantum devices based on various physical effects. The first experiments with laser pumped cesium atoms allowed to reach almost $100 \%$ polarization of alkali metal atoms in the magneto-dipole ultra-fine transition of ground state. An interesting continuation of such research were the precision measurement of the resonance frequency variation on $0-0$ transition caused by

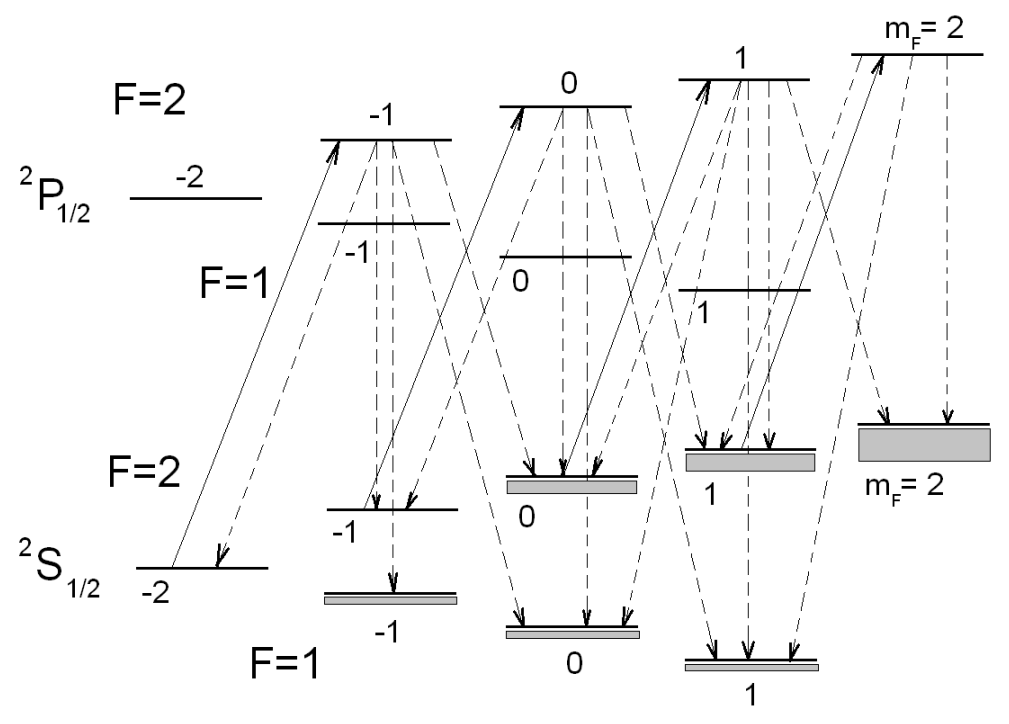

Fig. 1. The scheme of optical orientation of ${ }^{87} \mathrm{Rb}$ atoms in the ground state in terms of electrodipole transitions by $\sigma^{+}$polarized laser light of $D_{1}$ line. Solid arrows indicate direct transitions, dashed arrows indicate spontaneous transitions. 


\section{S. V. Ermak et al.}

the dynamic Stark effect offsets the energy sublevels of the atoms in the pumping radiation field. ${ }^{7,8}$ The method of optical pumping is the optical orientation. ${ }^{1}$ The energetic structure of ${ }^{87} \mathrm{Rb}$ atoms ground state $\left({ }^{2} S_{1 / 2}\right)$, first excited state $\left({ }^{2} P_{1 / 2}\right)$ and electro-dipole transitions by circularly polarized light $\left(\sigma^{+}\right.$polarization $)$is presented in Fig. 1. Optical orientation by circularly polarized radiation, where, as a result, the implementation of the magnetic quantum state selection rules depletes the number of magnetic sublevels with extreme value projection of a full momentum. Non-equilibrium population is created on a magnetic quantum sublevels. This method is used in quantum magnetometers.

\section{The System of Two Quantum Magnetometers}

The basis of the research experimental part was the magnetic induction meter scheme based on the system of two quantum magnetometers with optical pumping (SQMOP), one of which is self-oscillating low frequency (LF) magnetometer, another is the passive Z-magnetometer based on the UHF radio-optical resonance, which resonance frequency corresponds to magneto-dependent transitions between UFS sublevels with extreme value of a magnetic quantum number. The simplified functional scheme of such device is presented in Fig. 2.

The functional schemes of LF self-oscillating and UHF Z-type magnetometers is presented in Figs. 3 and 4, respectively. New result of this research is the use one

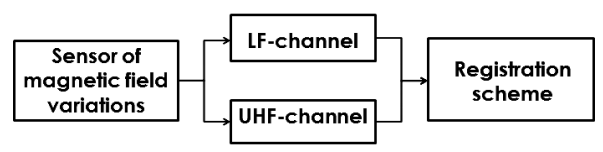

Fig. 2. Functional scheme of a two-channel system of quantum magnetometers with optical pumping.

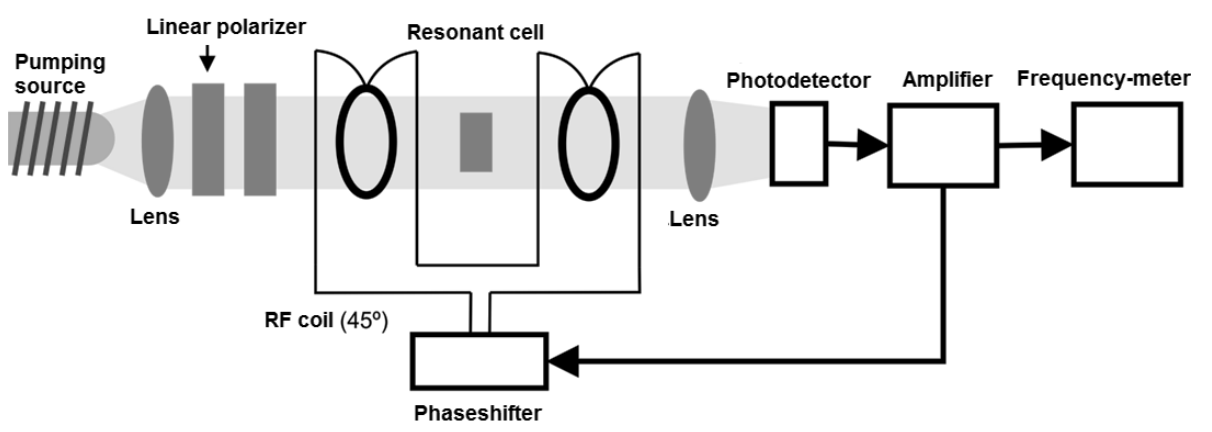

Fig. 3. Self-oscillating magnetometer, LF-channel. 


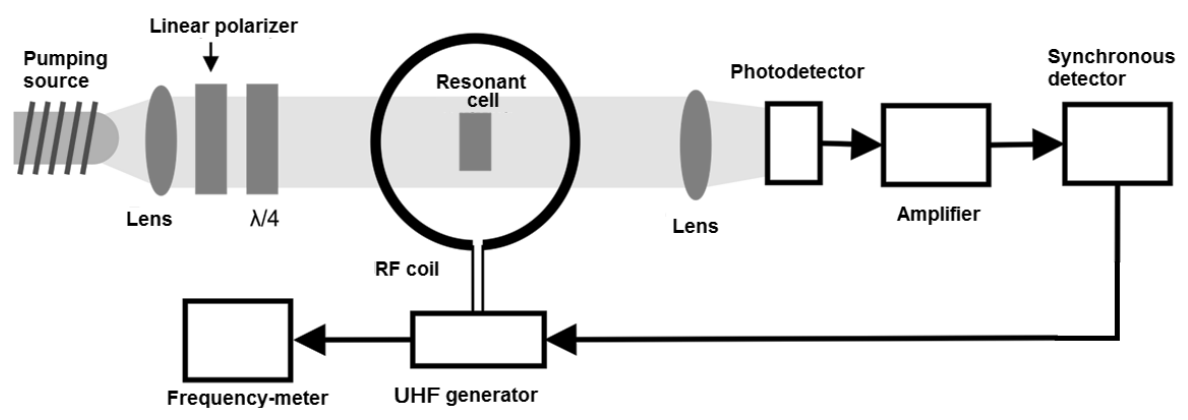

Fig. 4. Z - magnetometer, UHF-channel.

cell with anti-relaxation coating, containing alkali atoms for two magnetometers, that provides additional possibilities in the light shift reduction.

Experimental verification of the light shift compensation effect was carried out in the context of the ${ }^{87} \mathrm{Rb}$ atoms vapors laser optical pumping of ground state $\mathrm{F}=2$ by light component $\mathrm{S}_{1 / 2}-\mathrm{P}_{1 / 2}$ of $\mathrm{D}_{2}$ line in SQMOP. The functional scheme of SQMOP contains known elements used in gas-cell atomic clock. The scheme is operated on the UHF radio-optical resonance signals and on the weighted average resonance frequency of the Zeeman structure.

This scheme provides simultaneous measurement of magnetic field fluctuations by registration of the UHF measuring channel error signal and by measurement of the LF measuring channel signal frequency, which react to changing of an external magnetic field and laser pumping parameters. Then, two channels signals are detected by the registration scheme where they are mutually subtracted (see Fig. 5). This method provides the magnetic fluctuations and light shift components compensation. $^{4}$

Further, Allan variance for the differential signal of the two quantum magnetometers was calculated by means of the Matlab program.

In Fig. 6, Allan variance is presented for two directions of pumping light circular polarization, $\left(\sigma^{+}\right.$or $\left.\sigma^{-}\right)$with light power of $\sim 100 \mu \mathrm{W}$, that corresponds to the quality factor maximum mode $(\mathrm{QFM})$ of $\mathrm{LF}$ and $\mathrm{UHF}$ radio optical resonance signals. The represented experiments are provided under other identical conditions.

Thus, there is a principal possibility of scalar and vector light shift mutual compensation by choosing a suitable sign of the pumping light circular polarization, that allows to realize the reduced resonance frequency light shift of the atomic clock based on the two quantum magnetometers system.

The greatest interest in applications represents the area of averaging time from $10^{2}$ till $10^{3}$ seconds. It was the cause to carry out a number of experiments for the quantum magnetometers operating mode optimization in time of averaging exceeding a time barrier in hundreds of seconds. ${ }^{9}$ It was found experimentally, that this 


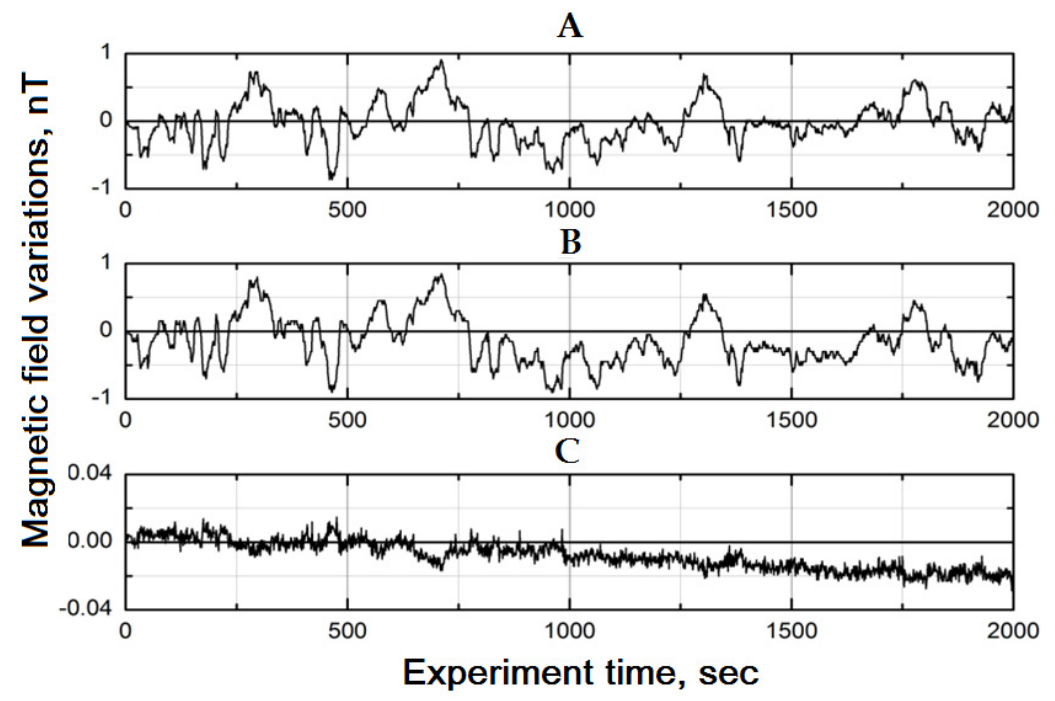

Fig. 5. The magnetic fluctuations compensation in the system of two quantum magnetometers. A - LF-channel, B - UHF-channel, C - difference of the magnetic variations registered by LF and UHF channels.

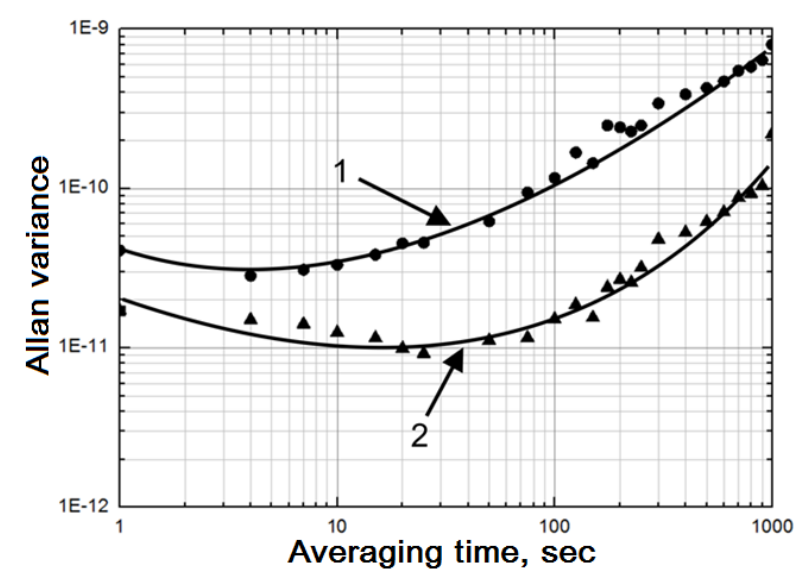

Fig. 6. Allan variance for system of two quantum magnetometers. Graphs 1 and 2 corresponds the polarization of pumping light of $\sigma^{+}$and $\sigma^{-}$types.

variance limit corresponds to a certain intensity of the pumping light (see Fig. 7). The intensity in this case was $\sim 25 \mu \mathrm{W}$ under $\sigma^{+}$polarization of optical pumping. These conditions correspond to the mode of maximal long-term stability (MLTS).

In the same figure similar dependencies for SQMOP are given in QFM mode (dependence 1) and for atomic clock on 0-0 transition (dependence 3). Short-term 


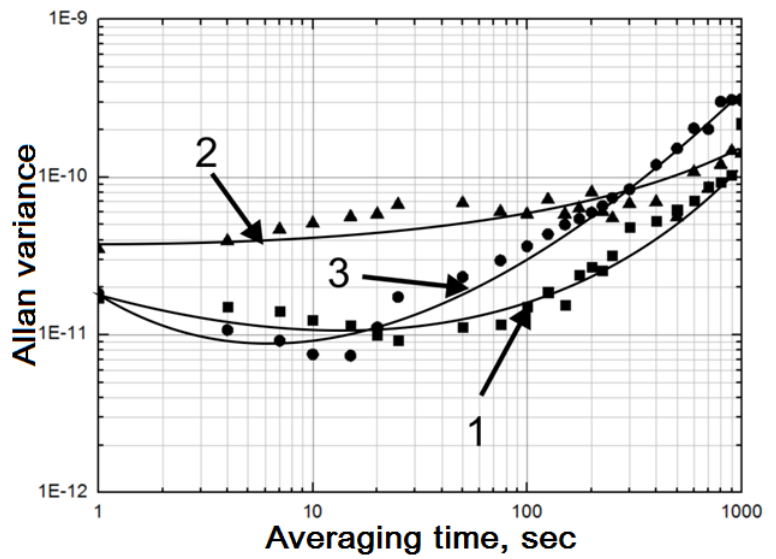

Fig. 7. Allan variance for the system of two quantum magnetometers. Graphs 1 and 2 corresponds to laser power of $\sim 100 \mu \mathrm{W}$ (QFM mode) and $\sim 25 \mu \mathrm{W}$ (MLTS mode) under $\sigma^{+}$polarization of optical pumping, graph 3 Allan variance for atomic clock on 0-0 transition.

stability of SQMOP in the MLTS mode (see Fig. 7) significantly concedes to similar parameter during the operation of the device in the MQF mode that is explained by decrease in a quality factor of observed signals because there is discrepancy in intensity of pumping to its optimum level within a flicker floor. However, at long operation of the device in MLTS mode is justified by more flat dependence of Allan variance with time growth, that in the conditions of the light shift component compensation allows to improve the SQMOP precision properties. In the MQF mode a flicker floor of SQMOP and atomic clock on 0-0 transition within several tens seconds of supervision practically coincide, however, the advance in area of long-term stability in Fig. 7 shows undoubted advantage of the studied scheme in comparison with atomic clock on 0-0 transition.

Thus, the correct choice of the laser pumping light polarization allows light shift various component mutual compensation of the atomic clock resonant frequency and therefore, increases its long-term stability.

\section{Summary}

It is shown, experimentally, that the effect of the light shift components compensation allows to reduce the frequency Allan variance of the atomic clock based on the system of two quantum magnetometers in comparison with the atomic clock on $0-0$ transition.

\section{References}

1. W. Happer, Rev. Mod. Phys. 44, 170 (1972).

2. F. Riehle, Frequency Standards: Basics and Applications (Wiley, Weinheim, 2005).

3. W. Happer and B. S. Mathur, Phys. Rev. 163, 12 (1967). 
4. A. A. Baranov, S. V. Ermak, E. A. Sagitov, R. V. Smolin and V. V. Semenov, Journal of Experimental and Theoretical Phys. 121, 393 (2015).

5. Y.-Y. Jau, A. B. Post, N. N. Kuzma, A. M. Braun, M. V. Romalis and W. Happer, Phys. Rev. Lett. 92, 1 (2004).

6. S. Siahatgor and V. E. Hochuli, Quantum Electron. 5, 295 (1969).

7. M. Arditi and J. L. Picque, Optics Comm. 15, 317 (1975).

8. M. Arditi and J. L. Picque, Atom. Phys. 88, 331 (1975).

9. E. B. Aleksandrov and A. K. Vershovskiy, Usp. Fiz. Nauk 179, 605 (2009), in Russian. 